\title{
Three-Dimensional Eye Shape, Myopic Maculopathy, and Visual Acuity: The Zhongshan Ophthalmic Center-Brien Holden Vision Institute High Myopia Cohort Study
}

Xinxing Guo, MD, PhD, ${ }^{1, *}$ Ou Xiao, MD, PhD, ${ }^{1, *}$ Yanxian Chen, $\mathrm{MD},{ }^{1}$ Huawang $\mathrm{Wu}, \mathrm{MD},{ }^{2}$ Linxing Chen, $\mathrm{MD},{ }^{1}$ Ian G. Morgan, $\mathrm{PhD},{ }^{1,3}$ Mingguang $\mathrm{He}, \mathrm{MD}, \mathrm{PhD}^{1,4}$

Purpose: To investigate the relationship among eye shape, myopic maculopathy, and visual acuity in highly myopic eyes using 3-dimensional (3D) magnetic resonance imaging (MRI).

Design: Observational case series.

Participants: The study included 190 eyes of 95 participants with bilateral high myopia from the Zhongshan Ophthalmic Center-Brien Holden Vision Institute High Myopia Cohort Study, with spherical power $\leq-6.00$ diopters (D) in both eyes.

Methods: The participants underwent best-corrected visual acuity (BCVA), fundus photography, cycloplegic refraction, and ocular biometry. Posterior staphyloma was identified with 3D MRI, and ocular shapes were categorized into spheroidal, ellipsoidal, conical, nasally distorted, temporally distorted, and barrel shapes according to the inferior view from T2-weighted 3D MRI (Achieva 3.0T; Philips Medical Systems, Best, the Netherlands). Myopic maculopathy was graded as C0 to C4 according to the International Photographic Classification and Grading System using fundus photography. The statistical significance of the differences in distribution of myopic maculopathy and BCVA in eyes with different ocular shapes was determined using Pearson's chi-square tests.

Main Outcome Measures: Distributions of myopic maculopathy and BCVA in relation to different eye shapes.

Results: The mean spherical equivalent and axial length were $-11.74 \pm 4.10 \mathrm{D}$ and $28.18 \pm 1.73 \mathrm{~mm}$ in the right eyes, respectively. The same ocular shapes were observed in both eyes in 69 participants $(72.6 \%)$. The predominant shape was spheroidal (53.7\%), followed by nasally distorted and conical types (both 14.7\%). C2 and above myopic maculopathy was observed in all barrel-shaped eyes, in $75 \%$ temporally distorted eyes, and in $71.4 \%$ nasally distorted and conical eyes. In eyes with posterior staphyloma $(n=22), 18(81.8 \%)$ had $\mathrm{C} 2$ and above myopic maculopathy compared with 40 eyes $(54.8 \%)$ without posterior staphyloma $(n=73)$. Eyes with temporal and nasal distortion, and eyes with staphyloma were more likely to have BCVA $<20 / 40$.

Conclusions: Not all highly myopic eyes are deformed. Spheroid was the predominant ocular shape in this series of young patients with high myopia bilaterally. Barrel-shaped and temporally distorted eyes present significant myopic maculopathy, whereas eyes with posterior staphyloma display more severe chorioretinal atrophy. Eyes of more deformed shapes tend to have more severe myopic maculopathy and worse BCVA. Ophthalmology 2017;124:679687 @ 2017 by the American Academy of Ophthalmology

Twenty-one percent of Taiwanese high school graduates were reported to be highly myopic in 2000, and the prevalence of high myopia has increased dramatically over the past few decades in other regions where myopia has become epidemic. $^{1,2}$ It is estimated that there will be approximately 1 billion high myopes ( $>5$ diopters [D]) globally by $2050 .{ }^{3}$ People with high myopia are at increased risk of various ocular complications such as glaucoma, cataract, and retinal detachment, as well as myopic maculopathy and posterior staphyloma. ${ }^{4-6}$ Various terms have been used to describe macular pathologic changes in myopia, including myopic maculopathy, myopic macular degeneration, and myopic retinopathy. In this study, we endorse the term myopic maculopathy as proposed in a previous consensus. In highly myopic eyes, myopic maculopathy is associated with more myopic spherical equivalent refraction (SER), greater axial elongation, and deformed ocular shapes, causing deterioration of vision. ${ }^{8,9}$ However, early findings primarily characterized eye shape in terms of imaging using ultrasound or optical coherence tomography, which provided only limited information about the 3-dimensional (3D) structure of the eye. ${ }^{10-13}$

Since its first application in determining the contours and volumes of eyes in the $1990 \mathrm{~s},{ }^{14}$ the technique of magnetic 
resonance imaging (MRI) has proved to be very useful in defining ocular shapes. ${ }^{15,16}$ With the improvement of high-resolution imaging and the development of 3D reconstruction technology, high-resolution 3D MRI for characterizing ocular shapes can now provide more detailed information about highly myopic eyes, especially those with posterior staphyloma. ${ }^{17,18}$ The technique has become a unique tool for viewing stretching and curvature changes, both locally and globally across the eye.

In 2011, Moriyama et $\mathrm{al}^{17}$ first reported topographic findings in highly myopic eyes using high-resolution 3D MRI. The authors reported ocular shapes in 44 patients with extreme myopia of refractive error $\geq-8.00 \mathrm{D}$ or with axial length (AL) $>26.5 \mathrm{~mm}$. Eye shapes were categorized into 4 distinct groups: nasally distorted, temporally distorted, cylindrical, and barrel type. The correlations of myopic fundus lesions and types of ocular shape were investigated in 30 patients with the same ocular shape in both eyes. Myopic choroidal neovascularization, chorioretinal atrophy, and myopic tractional maculopathy ${ }^{5}$ were not found to be associated with ocular shape in the series. The sample size was relatively small in the study, and at that stage, there was no standard grading system for the classification of fundus lesions. In a more recent study, Moriyama et $\mathrm{al}^{19}$ performed quantitative analysis of 3D MRI obtained in 117 patients, but neither fundus changes nor visual acuity was assessed.

This study explores the associations between ocular shape, including the presence of posterior staphyloma, characterized with high-resolution MRI, myopic maculopathy graded according to the recently developed International Photographic Classification and Grading System, and best-corrected visual acuity (BCVA) in a group of bilateral high myopes.

\section{Methods}

\section{Study Participants}

Participants were a subsample from the Zhongshan Ophthalmic Center-Brien Holden Vision Institute (ZOC-BHVI) Guangzhou High Myopia Cohort Study. Written informed consent was obtained from participants for both the cohort study and the additional MRI scanning. The project conformed to the tenets of the Declaration of Helsinki, and ethical approval was obtained from the Institutional Review Board of Zhongshan Ophthalmic Center.

The ZOC-BHVI Guangzhou High Myopia Cohort Study has been described in detail (Chen Y, Xiao O, Guo X, etc. Methodology of the ZOC-BHVI High Myopia Cohort Study: the Onset and Progression of Myopic Pathologies and Associated Risk Factors in Highly Myopic Chinese). Briefly, participants aged 7 to 70 years with bilateral high myopia, defined as no less than $-6.00 \mathrm{D}$ spherical power in both eyes and no secondary causes of myopia, any history of refractive or intraocular procedures including vitreoretinal surgeries, or any severe systemic conditions, were enrolled from the optometry clinic at Zhongshan Ophthalmic Center. For the current MRI study, a $10 \%$ subsample was drawn from the general cohort using stratified random sampling into 2 age strata, aged $<40$ and $\geq 40$ years, after excluding those with contradictions to MRI scanning, such as a heart pacemaker or metallic foreign body implant. A total of 100 participants (75 aged $<40$ years and 25 aged $\geq 40$ years) were initially invited to the current study. Among these, 95 subjects $(95.0 \%)$ consented to further examination with MRI scanning.

\section{Three-Dimensional Magnetic Resonance Imaging Analysis}

The participants were referred to the Guangzhou Brain Hospital for turbo spin-echo T2-weighted MRI using a whole-body scanner (Achieva 3.0T; Philips Medical Systems, Best, the Netherlands) with an 8-channel phased-array head coil. The participants were instructed to keep both eyes closed with minimal movement during the scanning. Scanning sequences (repetition time $=2500 \mathrm{~ms}$; echo time $=248 \mathrm{~ms}$; flip angle $=90^{\circ}$; field of view $=256 \times 256 \times 188 \mathrm{~mm}$ ) were performed with maximum water-fat shift. The image resolution was $1 \times 1 \times 1 \mathrm{~mm}$. The scanning time for each participant was approximately 10 minutes. Volume renderings of the images were generated from highresolution 3D data on a computer workstation (OsiriX 7.0; OsiriX Medical Image Software, Bernex, Switzerland) with manual segmentation to delineate the right and left eyes in the T2-weighted image.

\section{Types of Ocular Shapes in Highly Myopic Eyes}

Ocular shapes were identified dynamically from the rotating view both horizontally and vertically. An eye was first categorized into spheroidal or nonspheroidal from the rotating 3D view. Then, for eyes classified as nonspheroidal, inferior and nasal views were further analyzed. Symmetry of the posterior segment curvature was assessed, followed by analysis of changes in the curvature of the posterior surface. Staphyloma was defined as an outpouching from the wall of the eye with a radius of curvature less than that of the surrounding areas of the posterior segment. ${ }^{20}$ The number of protrusions was identified from the rotating 3D view. Two graders (X.G., Y.C.) masked to the refraction data independently graded the ocular shapes, with agreement of 0.84 for the ocular shape and 0.86 for the posterior staphyloma. Disagreement was adjudicated by a third grader (O.X.).

The inferior view of the eye, which best displayed the contour profile and the symmetry between the nasal and temporal posterior segment, was used to evaluate the ocular shape. A total of 6 distinct categories of eye shape were defined, as shown in Figure 1:

(1) Spheroidal: nasal and temporal symmetry without obvious elongation of the posterior segment or change in the radius of curvature

(2) Ellipsoidal: nasal and temporal symmetry with elongated posterior segment and no change in the radius of curvature

(3) Conical: nasal and temporal symmetry with elongated posterior segment and equally steeper than a circle radius of curvature; specifically, this category was comparable to the "cylindrical" type proposed by previous studies ${ }^{16,18}$

(4) Nasally distorted: nasal and temporal asymmetry with elongated posterior segment and more protruded nasal half

(5) Temporally distorted: nasal and temporal asymmetry with elongated posterior segment and more protruded temporal half

(6) Barrel-shaped: nasal and temporal symmetry with elongated posterior segment and flatter than a circle radius of curvature

In this study, conical, nasally distorted, temporally distorted, and barrel-shaped eyes were considered to be ocular deformities. 


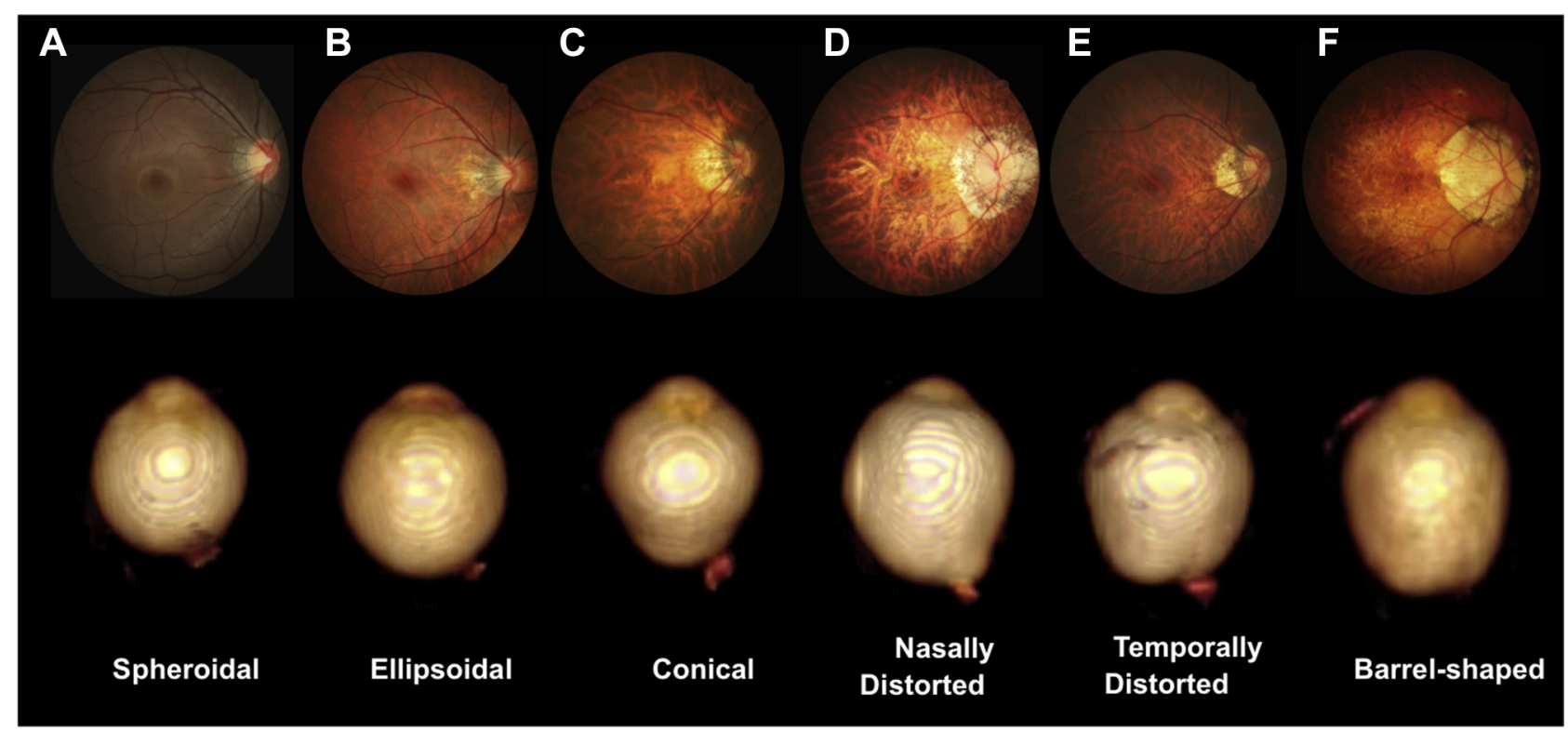

Figure 1. Myopic maculopathy in highly myopic right eyes of different shapes identified from the inferior view of 3-dimensional (3D) magnetic resonance imaging (MRI). Ocular shapes were classified into 6 types: (A) spheroidal: nasal and temporal symmetry with a spheroidal posterior segment, where the macula showed mostly unremarkable (C0) or tessellated fundus (C1); (B) ellipsoidal: nasal and temporal symmetry with an elongated posterior segment and no obvious change of radius of curvature, where the macula showed mostly tessellated fundus (C1) or diffuse chorioretinal atrophy (C2); (C) conical: nasal and temporal symmetry with an elongated posterior segment and equally steeper than a circle radius of curvature, where the macula showed mostly tessellated fundus (C1) or diffuse chorioretinal atrophy (C2); (D) nasally distorted: nasal and temporal asymmetry with an elongated posterior segment and more protruded nasal half, where the macula showed mostly diffuse chorioretinal atrophy (C2); (E) temporally distorted: nasal and temporal asymmetry with an elongated posterior segment and more protruded temporal half, where the macula showed mostly diffuse chorioretinal atrophy (C2); (F) barrel-shaped: nasal and temporal symmetry with an elongated posterior segment and flatter than a circle radius of curvature, where the macula showed mostly diffuse chorioretinal atrophy (C2).

\section{Ophthalmic Examinations}

All of the participants underwent a series of ophthalmic evaluations. The BCVA was tested using a retroilluminated logarithm of the minimum angle of resolution chart with tumbling-E optotypes (Precision Vision, La Salle, IL). Axial length was measured with optical low-coherence reflectometry (Lenstar LS900; Haag-Streit AG, Koeniz, Switzerland) and with partial coherence interferometry (IOL Master; Carl Zeiss Meditec, Oberkochen, Germany) when AL exceeded the Lenstar measurement range (up to $32 \mathrm{~mm}$ ). Refraction data were obtained with an autorefractor (KR8800; Topcon, Tokyo, Japan) after complete cycloplegia for both eyes, determined by the absence of a light reflex and a dilated pupil at least $6 \mathrm{~mm}$ in diameter.

\section{Fundus Color Photograph Grading}

Two $45^{\circ}$ fundus color photographs, centered respectively at the optic nerve and macula, were obtained for each eye with fully dilated pupils after completion of other evaluations. Fundus photographs were graded according to the International Photographic Classification and Grading System scheme, ${ }^{7}$ which classifies myopic maculopathy into 5 categories of "no myopic retinal lesions" (C0), "tessellated fundus only" (C1), "diffuse chorioretinal atrophy" (C2), "patchy chorioretinal atrophy" (C3), and "macular atrophy" (C4). Plus lesions including lacquer cracks, myopic choroidal neovascularization, and Fuchs' spot were also identified.

\section{Statistical Analysis}

Refractive error was determined by SER, calculated as spherical power $+1 / 2$ cylindrical power. Age was categorized into $<40$ and $\geq 40$ years; $\mathrm{AL}$ was classified into 3 categories: $<28.00$, 28.00 30.00, and $\geq 30.00 \mathrm{~mm}$; and SER was classified into 3 categories: $-6.00 \sim-10.00,-10.00 \sim-15.00$, and $\leq-15.00 \mathrm{D}$. The BCVA was categorized into $\geq 20 / 40$ and $<20 / 40$. The distributions of various ocular shapes by the age, AL, and SER categories were analyzed by Pearson chi-square tests, as were the distributions of BCVA with the different eye shapes identified from MRI. Multiple logistic regression analysis was used to assess the odds ratio (OR) of risk factors for distorted ocular shapes. Distributions of different myopic maculopathy grades by ocular shape are presented as stacked bar graphs. A $P$ value $<0.05$ was considered statistically significant.

\section{Results}

A total of 95 participants (190 eyes) were studied, among which 49 $(51.6 \%)$ were male and $46(48.4 \%)$ were female, with a mean age of 32.0 \pm 14.0 years (range, $12.0 \sim 67.0$ years) and no significant age difference between genders $(P=0.556)$. Because the 2 eyes of the same individual were highly correlated, only data for the right eyes are presented. Mean SER was $-11.74 \pm 4.10 \mathrm{D}$, and mean AL was $28.18 \pm 1.73 \mathrm{~mm}$. The BCVA ranged from 20/2000 to 20/16, with a median of $20 / 20$.

\section{Classification of Magnetic Resonance Imaging Eye Shapes in High Myopia}

Distributions for the ocular shapes of the right eyes are presented in Table 1. Of the 95 right eyes scanned, spheroidal shape was observed in 51 eyes $(53.7 \%)$, ellipsoidal shape was observed in 8 eyes $(8.4 \%)$, conical shape was observed in 14 eyes $(14.7 \%)$, nasally distorted 
Table 1. Ocular Characteristics in Highly Myopic Eyes with Different Shapes Based on 3-Dimensional Magnetic Resonance Imaging

\begin{tabular}{|c|c|c|c|c|c|c|c|c|}
\hline & Total Eyes, no. & Spheroidal & Ellipsoidal & Conical & $\begin{array}{c}\text { Nasally } \\
\text { Distorted }\end{array}$ & $\begin{array}{l}\text { Temporally } \\
\text { Distorted }\end{array}$ & Barrel-shaped & $P$ Value \\
\hline No. of eyes & 95 & 51 & 8 & 14 & 14 & 4 & 4 & \\
\hline Age (yrs) & & & & & & & & $<0.001$ \\
\hline$<40$ & 70 & $44(62.9)$ & $8(11.4)$ & $6(8.6)$ & $10(14.3)$ & $1(1.4)$ & $1(1.4)$ & \\
\hline$\geq 40$ & 25 & $7(28.0)$ & $0(0.0)$ & $8(32.0)$ & $4(16.0)$ & $3(12.0)$ & $3(12.0)$ & \\
\hline $\mathrm{AL}(\mathrm{mm})$ & & & & & & & & $<0.001$ \\
\hline$\sim<28.00$ & 45 & $37(82.2)$ & $1(2.2)$ & $4(8.9)$ & $1(2.2)$ & $2(4.4)$ & $0(0.0)$ & \\
\hline $28.00 \sim<30.00$ & 37 & $14(37.8)$ & $7(18.9)$ & $8(21.6)$ & $6(16.2)$ & $2(5.4)$ & $0(0.0)$ & \\
\hline$\sim \geq 30.00$ & 13 & $0(0.0)$ & $0(0.0)$ & $2(15.4)$ & $7(53.9)$ & $0(0.0)$ & $4(30.8)$ & \\
\hline Spherical equivalence (D) & & & & & & & & $<0.001$ \\
\hline$-10.00 \sim \leq-6.00$ & 38 & $30(79.0)$ & $2(5.3)$ & $3(7.9)$ & $1(2.6)$ & $2(5.3)$ & $0(0.0)$ & \\
\hline$-15.00 \sim \leq-10.00$ & 37 & $19(51.4)$ & $4(10.8)$ & $7(18.9)$ & $5(13.5)$ & $2(5.4)$ & $0(0.0)$ & \\
\hline$\sim \leq-15.00$ & 20 & $2(10.0)$ & $2(10.0)$ & $4(20.0)$ & $8(40.0)$ & $0(0.0)$ & $4(20.0)$ & \\
\hline Staphyloma on MRI & & & & & & & & $<0.001$ \\
\hline Without staphyloma & 73 & $51(69.9)$ & $8(11.0)$ & $4(5.5)$ & $7(9.6)$ & $2(2.7)$ & $1(1.4)$ & \\
\hline With staphyloma & 22 & $0(0.0)$ & $0(0.0)$ & $10(45.5)$ & $7(31.8)$ & $2(9.1)$ & $3(13.6)$ & \\
\hline
\end{tabular}

$\mathrm{AL}=$ axial length; $\mathrm{D}=$ diopters; $\mathrm{MRI}=$ magnetic resonance imaging.

Data are presented as $\mathrm{n}(\%)$ in each row unless otherwise indicated.

shaped was observed in 14 eyes (14.7\%), temporally distorted shape was observed in 4 eyes (4.2\%), and barrel shape was observed in 4 eyes $(4.2 \%)$. The corresponding numbers and proportions for the left eyes were $54(56.8 \%), 9(9.5 \%), 11(11.6 \%), 18(19.0 \%), 1$ $(1.1 \%)$, and $2(2.0 \%)$, respectively. In this sample, 69 participants $(72.6 \%)$ had the same ocular shape in both eyes.

Staphyloma was identified on MRI in 22 right eyes (46.3\%) and 17 left eyes (43.2\%). In 13 participants (13.7\%), staphyloma was identified on MRI in both eyes. Among the right eyes with staphyloma on MRI, 1 protrusion was observed in 20 cases $(90.9 \%)$, and 2 protrusions were found in 2 cases $(9.1 \%)$.

\section{Ocular Characteristics in Different Eye Shapes}

Ocular shape was associated with age and degree of high myopia suggested by AL and SER (Table 1). Spheroid was the predominating ocular shape for people younger than 40 years of age, and other ocular shapes tended to be more common in the older age group. Spheroidal and ellipsoidal shapes were consistently found in the less severe highly myopic eyes and in the eyes without staphyloma on MRI. In multiple logistic regression analysis in which ocular deformity was considered as the outcome variable and age, sex, and AL or SER were regarded as the independent variables, findings suggested that distorted ocular shapes (including conical, nasally distorted, temporally distorted, and barrel-shape) tended to be more common in the older age group (OR, 5.64; $P=0.002)$ and in the eyes with AL $28 \mathrm{~mm}$ or longer (OR, 5.86; $P=0.001)$, or eyes with SER $-10.00 \mathrm{D}$ or greater (OR, 3.12; $P=0.030$ ).

Staphyloma on MRI was more frequently observed in people with older age and in eyes with longer AL or more myopic SER (Table 2). In eyes with AL less than $28 \mathrm{~mm}$ or with SER less myopic than $-10.00 \mathrm{D}, 10 \%$ were found with staphyloma on MRI. Multiple regression analysis showed that staphyloma on MRI was more common in people 40 years of age and older (OR, 14.79; $P<0.001)$ and in eyes with AL $28 \mathrm{~mm}$ or longer (OR, 6.98; $P=0.005$ ) or eyes with SER $-10.00 \mathrm{D}$ or greater (OR, 4.92; $P=0.019)$.

\section{Myopic Maculopathy in Different Eye Shapes}

Fundus features in different eye shapes are displayed in Figure 1, and the distributions of myopic maculopathy with ocular shapes and staphyloma on MRI are shown in Figures 2 and 3, respectively. C2 and more severe myopic maculopathy grades were uncommon in eyes with a spheroidal shape, whereas more than $75 \%$ of the distorted eyes had $\mathrm{C} 2$ or greater myopic maculopathy grades.

There were cases in which the MRI findings and myopic maculopathy grading did not correspond. Two spheroidal eyes presented diffuse chorioretinal atrophy in Figure 4, whereas 1 nasally distorted and 1 conical eye presented minimal myopic maculopathy (Fig 5). More detailed evaluations revealed some underlying associations between these findings. C2 myopic maculopathy was found in 2 spheroidal eyes (Fig 4A, E), with mild diffuse chorioretinal atrophy along the temporal optic nerve, where our preliminary observations suggest most diffuse atrophy initially develops. Tessellated fundus with $\mathrm{C} 1$ myopic maculopathy was seen in 1 nasally distorted eye (Fig 5A) with peripapillary atrophy but little maculopathy. The mild deformity toward the optic nerve viewed nasally or inferiorly may explain such fundus features. $\mathrm{C} 0$ was classified in a conical eye with 1 protrusion of staphyloma on MRI (Fig 5E). The posterior staphyloma was observed in the center from the back view, but the nasal view revealed that the most protruded point was inferior to the optic nerve, corresponding to the tessellated changes seen around the inferior macular vascular arcade, along with the inferior-temporal rotation of the optic nerve head.

\section{Best-Corrected Visual Acuity in Eyes of Different Shapes}

Distributions of BCVA in eyes of different ocular shapes are shown in Table 3. A BCVA of 20/40 was used as a cutoff, and 83 eyes $(87.4 \%)$ had BCVA $\geq 20 / 40$. Spheroidal and ellipsoidal eyes had predominately normal vision, whereas the poorest vision was observed among nasally and temporally distorted eyes. Half of the eyes with staphyloma on MRI had compromised vision.

\section{Discussion}

More than $50 \%$ of eyes displayed a spheroidal shape on highresolution 3D MRI in this group of bilateral high myopes. The proportion was higher in participants younger than 40 
Table 2. Ocular Characteristics in Highly Myopic Eyes with and without Posterior Staphyloma Based on 3-Dimensional Magnetic Resonance Imaging*

\begin{tabular}{|c|c|c|c|c|}
\hline & $\begin{array}{l}\text { Total } \\
\text { Eyes, } \\
\text { no. }\end{array}$ & $\begin{array}{l}\text { Eyes without } \\
\text { Staphyloma } \\
\text { on MRI }\end{array}$ & $\begin{array}{l}\text { Eyes with } \\
\text { Staphyloma } \\
\text { on MRI }\end{array}$ & $\begin{array}{c}P \\
\text { Value }\end{array}$ \\
\hline No. of eyes & 95 & 73 & 22 & \\
\hline Age (yrs) & & & & $<0.001$ \\
\hline$<40$ & 70 & $62(88.6)$ & $8(11.4)$ & \\
\hline$\geq 40$ & 25 & $11(44.0)$ & $14(56.0)$ & \\
\hline $\mathrm{AL}^{-}(\mathrm{mm})$ & & & & $<0.001$ \\
\hline$\sim<28.00$ & 45 & $40(88.9)$ & $5(11.1)$ & \\
\hline $28.00 \sim<30.00$ & 37 & $30(81.8)$ & $7(18.9)$ & \\
\hline$\sim \geq 30.00$ & 13 & $3(23.1)$ & $10(76.9)$ & \\
\hline Spherical equivalence (D) & & & & $<0.001$ \\
\hline$-10.00 \sim \leq-6.00$ & 38 & $34(89.5)$ & $4(10.5)$ & \\
\hline$-15.00 \sim \leq-10.00$ & 37 & $32(86.5)$ & $5(13.5)$ & \\
\hline$\sim \leq-15 . \overline{0}$ & 20 & $7(35.0)$ & $13(65.0)$ & \\
\hline
\end{tabular}

$\mathrm{AL}=$ axial length; $\mathrm{D}=$ diopters; $\mathrm{MRI}=$ magnetic resonance imaging. *Data are presented as $\mathrm{n}(\%)$ in each row unless otherwise indicated.

years of age than in those older than 40 years. Ocular deformity was more prevalent in participants with older age, longer ocular AL, and more myopic SER. Among the deformed eyes, nasal distortion with protrusion around the optic nerve and conical contour were more frequently observed; barrel-shaped and temporally distorted eyes showed significant myopic maculopathy, whereas eyes with nasal and temporal distortions or with staphyloma on MRI presented worse BCVA. Different ocular shapes were associated with different ocular biometry and refractive characteristics, which indicates the involvement of various shape development patterns in the progression of high myopia.

The technique of MRI has been used to evaluate ocular shapes in developing eyes and eyes with different refractive

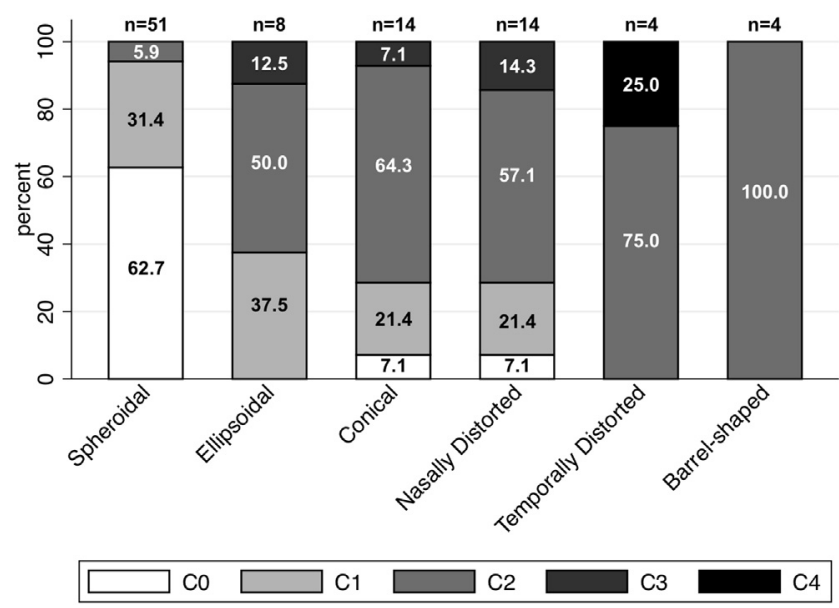

Figure 2. Proportions of myopic maculopathy by different ocular shapes identified from the inferior view of 3-dimensional magnetic resonance imaging. Because of the limitation of sample size, only 4 eyes were identified as temporally distorted and another 4 were identified as barrel-shaped. Only 1 temporally distorted eye was found to have C4 myopic maculopathy.

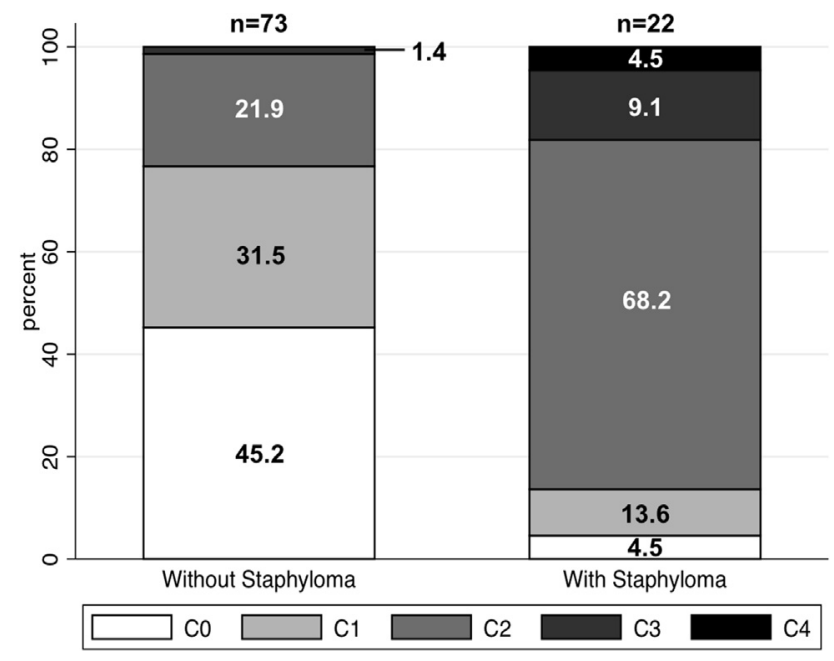

Figure 3. Proportions of myopic maculopathy by staphyloma identified from the inferior and rotating view of 3-dimensional magnetic resonance imaging. Greater proportions of more severe myopic maculopathy were seen in eyes with staphyloma.

status. With 2-dimensional MRI, it has been observed that infants were born with more oblate eyes, and during emmetropization, eyes became more prolate. ${ }^{21,22}$ In adult eyes, however, considerable variations have been observed. Cheng et $\mathrm{al}^{14}$ found no difference in the spheroelliptical shapes of hyperopic, emmetropic, and myopic eyes of up to -9.50 D. However, Atchison et $\mathrm{al}^{23}$ described emmetropic eyes as oblate ellipsoids and myopic eyes (up to $-12 \mathrm{D})$ as less oblate ellipsoids with increased axial, vertical, and horizontal dimensions. Gilmartin et $\mathrm{al}^{24}$ also reported that although myopic eyes were longer than emmetropic eyes with an oblate posterior chamber shape, they presented a lesser degree of oblate and were approximate to spheres. In the current qualitative study, more than half of the highly myopic eyes were identified as spheroidal and ellipsoidal, which were similar to those observed in emmetropic eyes. Thus, we defined ocular distortion in eyes with conical, nasally distorted, temporally distorted, and barrel shapes.

To the best of our knowledge, this study is among the first to report distributions of myopic fundus features and visual acuity in different eye shapes with or without posterior staphyloma identified with 3D MRI in highly myopic eyes. Limited ophthalmic assessment has been conducted with this imaging technique. The first observation of Moriyama et $\mathrm{al}^{17}$ noting more severe myopic cases showed that reconstructed 3D MRI scans allowed comprehensive ocular shape examinations, but only nasal distortion, temporal distortion, cylindrical, and barrel shapes were identified. Our sample, with younger and less myopic participants, showed additional categories of ocular shapes, including spheroidal and ellipsoidal; both were associated with less severe myopic maculopathy. Further quantitative analyses by Moriyama et $\mathrm{al}^{19}$ showed that up to $97.4 \%$ of eyes with pathologic myopia of at least $-8.00 \mathrm{D}$ were deformed. This study also confirmed the finding of more frequent chorioretinal changes and worse BCVA in eyes 

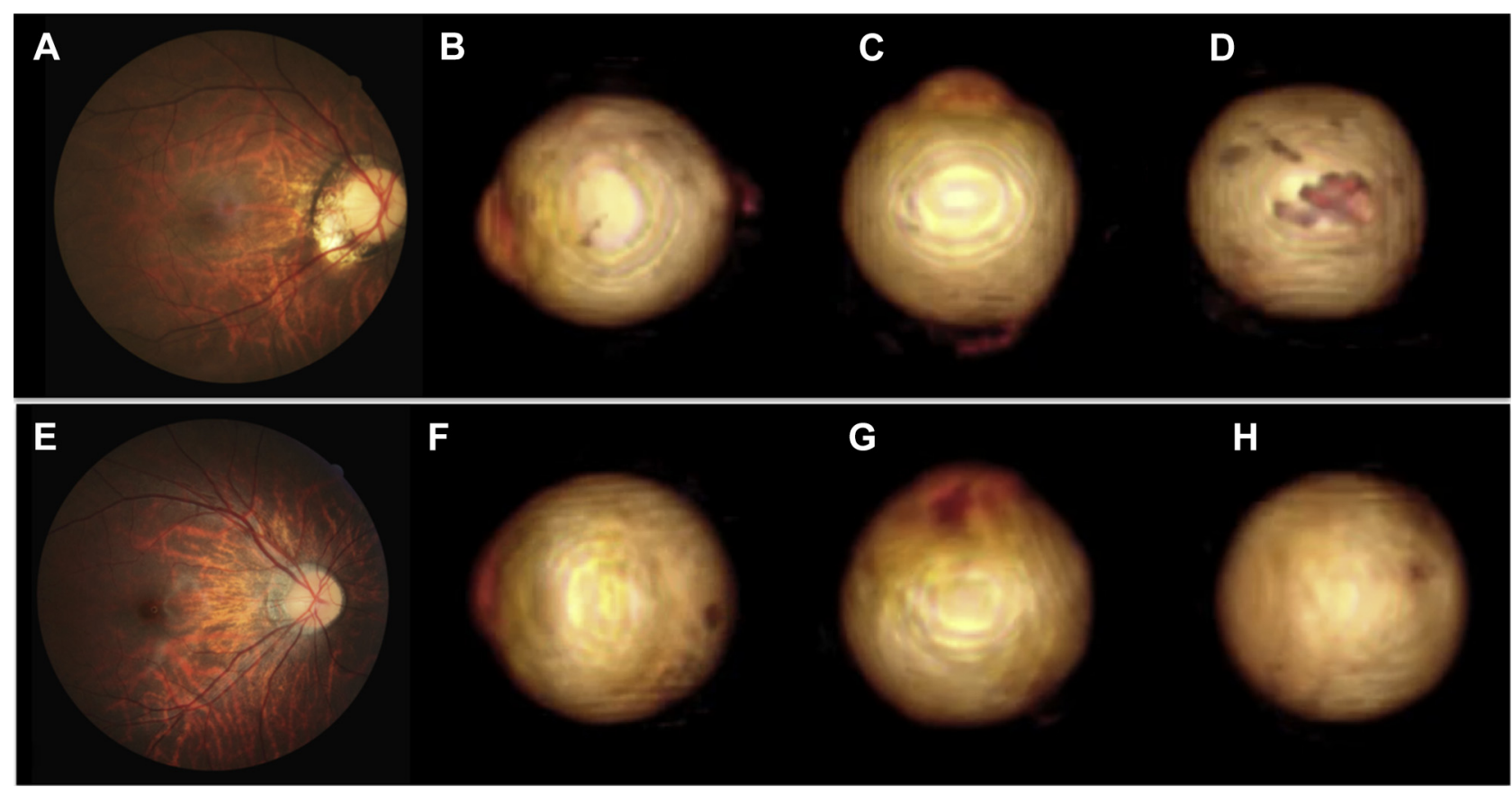

Figure 4. Highly myopic eyes with spheroidal ocular shapes and myopic maculopathy. A-D, Right eye of a 40-year-old woman with a refractive error of -11.50 diopters (D) and an axial length (AL) of $28.92 \mathrm{~mm}$. The fundus image shows peripapillary atrophy (PPA) and diffuse chorioretinal atrophy adjacent to the temporal PPA with C2 myopic maculopathy (A). B-D, Three-dimensional magnetic resonance imaging (MRI) scans of the globe. No obvious distortion or outpouching of the posterior segment is seen from nasal (B), inferior (C), or posterior perspectives (D). E-H, Right eye of an 18-year-old man with a refractive error of $-10.25 \mathrm{D}$ and an $\mathrm{AL}$ of $27.81 \mathrm{~mm}$. The fundus image shows tessellated fundus and diffuse chorioretinal atrophy temporal to the optic disc with $\mathrm{C} 2$ myopic maculopathy (E). F-H, Three-dimensional MRI scans of the globe. No obvious distortion or outpouching of the posterior segment is seen from nasal $(\mathrm{F})$, inferior $(\mathrm{G})$, or posterior perspectives $(\mathrm{H})$.

with posterior staphyloma, as suggested in the previous study by Ohno-Matsui, ${ }^{25}$ which was descriptive and enrolled both eyes of 105 patients. However, valid evaluation of the distributions of different ocular shapes and the corresponding fundus changes was not possible because of the analysis of both eyes and their high correlations.

Increased AL has been regarded as a surrogate measure of expanded eyes in highly myopic eyes. The minimum right eye $\mathrm{AL}$ in participants $\geq 40$ years of age was 25.68 $\mathrm{mm}$, and the distribution of $\mathrm{AL}$ with refraction followed a pattern similar to that in those younger than 40 years, with no outliers, suggesting the axial refractive nature of this group of high myopes. It has been well documented that more advanced myopic maculopathy is associated with older age, more myopic refractive error, and more elongated AL. ${ }^{26,27}$ However, the reported associations between $\mathrm{AL}$ and posterior staphyloma have not been consistent in previous reports because longer eyes did not necessarily develop staphyloma. ${ }^{8,13,28,29}$ Only 2-dimensional information was obtained in these studies, and there has been a lack of assessment of 3D topography features. Analysis with swept-source optical coherence tomography has demonstrated associations between fundus shape and myopic complications, but observations were limited to a 9-mm radius of evaluation at the posterior pole. ${ }^{30}$ This study found that most eyes with spheroidal and ellipsoidal shapes had less advanced myopic maculopathy and better
BCVA. However, temporally distorted eyes present more stretching of the macular lesion, which may explain the higher proportion of more severe myopic maculopathy observed. It seems that eye shape is an important risk factor for posterior staphyloma because of the more frequently observed staphyloma in eyes with a conical, nasally distorted, or barrel shape.

The significantly higher proportions of nasally distorted and conical eye shapes may be elucidated with the following mechanisms. First, curvature changes and scleral thinning have been observed in highly myopic eyes with protrusions, ${ }^{31}$ and thus distortion is more likely to occur in locations with weak scleral support. Second, histologic studies have shown extreme thinning of peripapillary scleral flange and choroid in highly axially myopic eyes with maximal thinning at the posterior pole. ${ }^{32,33}$ Therefore, the nasal distortion is believed to be generated from the absence of Bruch's membrane and choroid in the peripapillary region and lack of support from the thin sclera around the optic nerve. Our finding is in accordance with the observation by Moriyama et $\mathrm{al}^{17}$ that $36.7 \%$ of bilateral high myopes presented nasal distortion.

\section{Study Limitations}

Limitations of the current study included potential sampling bias because the participants were recruited from a tertiary eye care hospital. However, differences between eye care in 

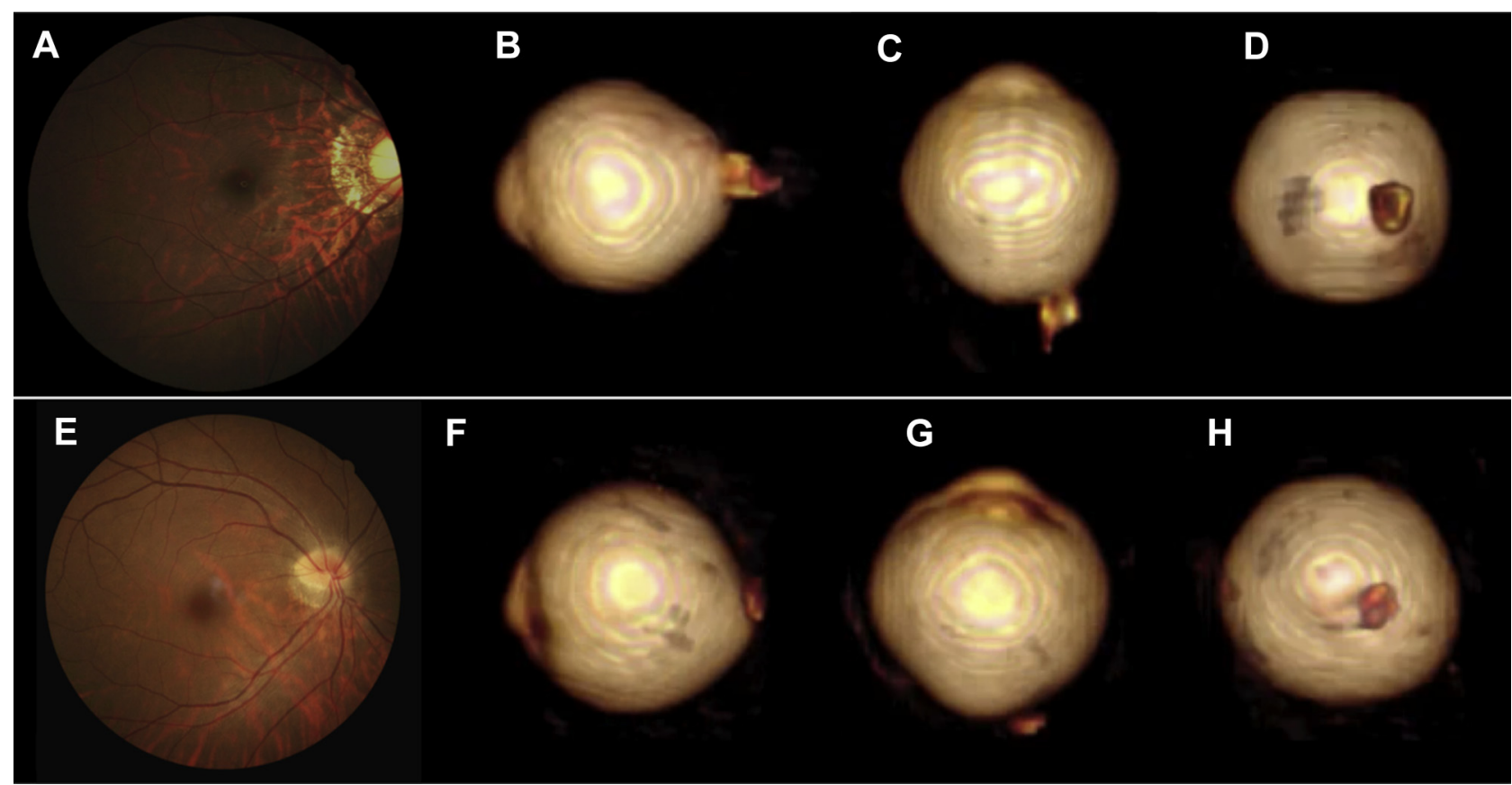

Figure 5. Highly myopic eyes with distorted ocular shapes and no myopic maculopathy. A-D, Right eye of a 23-year-old woman with a refractive error of -10.25 diopters (D) and an axial length (AL) of $28.36 \mathrm{~mm}$. The fundus image shows peripapillary atrophy (PPA) and tessellated changes along the macular arcade with C1 myopic fundus change (A). B-D, Three-dimensional magnetic resonance imaging (MRI) scans of the globe showed that the ocular shape was distorted. In the image viewed nasally, the maximally protruding part existed along the vertical axis (B). Nasal distortion of the posterior segment was seen from the interior perspective $(\mathbf{C})$ and no obvious protrusion was seen from the posterior perspective (D). E-H, Right eye of a 38 -year-old woman with a refractive error of $-9.63 \mathrm{D}$ and an $\mathrm{AL}$ of $26.43 \mathrm{~mm}$. The fundus image shows tessellated fundus change inferior to the macula with the optic disc tilted and rotated inferiorly-temporally. The fundus was graded as $\mathrm{CO}$ myopic change (E). F-H, Three-dimensional MRI scans of the globe showed distorted ocular shape with protrusion. In the image viewed nasally, the maximally protruding part existed inferior to the vertical axis $(\mathrm{F})$. Conical distortion of the posterior segment is seen from the inferior perspective $(\mathrm{G})$, and the posterior perspective shows the protrusion at the center of the posterior segment $(\mathrm{H})$.

China and more developed countries should be noted. Generally, patients in China go directly to a hospital or ophthalmic centers for refraction without referral, and private optometric practices are rare. The bilateral high myopes were referred from the general optometry clinic at

Table 3. Best-Corrected Visual Acuity in Highly Myopic Eyes with Different Shapes Based on Three-Dimensional Magnetic Resonance Imaging*

\begin{tabular}{lrccc}
\hline & \multirow{2}{*}{$\begin{array}{c}\text { Total } \\
\text { Eyes, }\end{array}$} & \multicolumn{2}{c}{ BCVA } & \\
Topographic Findings & no. & $\geq 20 / 40$ & $<20 / 40$ & \multirow{2}{*}{ P Value } \\
\hline No. of eyes (\%) & 95 & $83(87.4)$ & $12(12.6)$ & \\
Ocular shapes & & & & $<0.001$ \\
$\quad$ Spheroidal & 51 & $48(94.1)$ & $3(5.9)$ & \\
Ellipsoidal & 8 & $6(75.0)$ & $2(25.0)$ & \\
Conical & 14 & $14(100.0)$ & $0(0.0)$ & \\
$\quad$ Nasally distorted & 14 & $9(64.3)$ & $5(35.7)$ & \\
Temporally distorted & 4 & $2(50.0)$ & $2(50.0)$ & \\
$\quad$ Barrel-shaped & 4 & $4(100.0)$ & $0(0.0)$ & \\
Staphyloma on MRI & & & & $<0.001$ \\
$\quad$ Without staphyloma & 73 & $65(89.0)$ & $8(11.0)$ & \\
$\quad$ With staphyloma & 22 & $11(50.0)$ & $11(50.0)$ & \\
& & & &
\end{tabular}

$\mathrm{BCVA}=$ best-corrected visual acuity; $\mathrm{MRI}=$ magnetic resonance imaging. *Data are presented as $\mathrm{n}(\%)$ in each row unless otherwise indicated.
Zhongshan Ophthalmic Center, one of the leading eye care hospitals providing medical coverage for the entire area. All of the participants came in for simple refraction, with few cases of ocular complications, and thus are likely to be more representative of high myopes in the general Guangzhou population than a clinical sample assembled through a more specialized clinic would be. It is important to note that the eye shapes identified from the T2-weighted MRI actually represent the contour of the intraocular fluid rather than the external eye surface that is the sclera. Although the current study categorized highly myopic ocular shapes into 6 distinct types, few cases were identified with certain ocular shapes such as temporal distortion or barrel shape, which limits our knowledge of the associations between these ocular profiles and the fundus changes. The cross-sectional nature of the study also cannot define longitudinal changes in ocular shape. Two other limitations of our study should be noted. First, although we identified a subsample for MRI in subjects younger than 12 years of age, informed consent was not obtained for this group. Second, because of the way that the subjects were recruited, we did not have a sample of hyperopic and emmetropic eyes for analysis.

In conclusion, highly myopic eyes do not necessarily present ocular deformity, which is an important feature of pathologic myopia, especially in eyes with less severe myopia and shorter AL. Spheroid was the predominant 
ocular shape in this series of bilateral high myopes with minimal myopic maculopathy. Ellipsoidal shapes also were common. These shapes were more frequently seen in our sample than in those studied by Ohno-Matsui, ${ }^{34}$ and we suggest that they represent early stages in the appearance of eye distortion in the process of development of pathologic changes in highly myopic eyes. There is a general tendency for more severe myopic maculopathy and worse BCVA to be associated with more deformed eyes and those with staphyloma. We intend to follow this group of participants longitudinally to more precisely define changes in the shapes of highly myopic eyes and their causal links with myopic fundus features and deteriorating vision.

\section{References}

1. Lin LL, Shih YF, Hsiao CK, Chen CJ. Prevalence of myopia in Taiwanese schoolchildren: 1983 to 2000. Ann Acad Med Singapore. 2004;33:27-33.

2. Kim EC, Morgan IG, Kakizaki H, et al. Prevalence and risk factors for refractive errors: Korean National Health and Nutrition Examination Survey 2008-2011. PLoS One. 2013;8:e80361.

3. Holden BA, Fricke TR, Wilson DA, et al. Global prevalence of myopia and high myopia and temporal trends from 2000 through 2050. Ophthalmology. 2016;123:1036-1042.

4. Morgan IG, Ohno-Matsui K, Saw SM. Myopia. Lancet. 2012;379:1739-1748.

5. Ohno-Matsui K, Lai TY, Lai CC, Cheung CM. Updates of pathologic myopia. Prog Retin Eye Res. 2016;52:156-187.

6. Wong YL, Saw SM. Epidemiology of pathologic myopia in Asia and Worldwide. Asia Pac J Ophthalmol (Phila). 2016;5:394-402.

7. Ohno-Matsui K, Kawasaki R, Jonas JB, et al. International photographic classification and grading system for myopic maculopathy. Am J Ophthalmol. 2015;159:877-883.

8. Curtin BJ. The posterior staphyloma of pathologic myopia. Trans Am Ophthalmol Soc. 1977;75:67-86.

9. Chen H, Wen F, Li H, et al. The types and severity of high myopic maculopathy in Chinese patients. Ophthalmic Physiol Opt. 2012;32:60-67.

10. Curtin BJ, Karlin DB. Axial length measurements and fundus changes of the myopic eye. I. The posterior fundus. Trans Am Ophthalmol Soc. 1970;68:312-334.

11. Fledelius HC, Goldschmidt E. Oculometry findings in high myopia at adult age: considerations based on oculometric follow-up data over 28 years in a cohort-based Danish highmyopia series. Acta Ophthalmol. 2010;88:472-478.

12. Nakanishi H, Tsujikawa A, Gotoh N, et al. Macular complications on the border of an inferior staphyloma associated with tilted disc syndrome. Retina. 2008;28:1493-1501.

13. Hsiang HW, Ohno-Matsui K, Shimada N, et al. Clinical characteristics of posterior staphyloma in eyes with pathologic myopia. Am J Ophthalmol. 2008;146:102-110.

14. Cheng HM, Singh OS, Kwong KK, et al. Shape of the myopic eye as seen with high-resolution magnetic resonance imaging. Optom Vis Sci. 1992;69:698-701.

15. Singh KD, Logan NS, Gilmartin B. Three-dimensional modeling of the human eye based on magnetic resonance imaging. Invest Ophthalmol Vis Sci. 2006;47:2272-2279.
16. Atchison DA, Jones CE, Schmid KL, et al. Eye shape in emmetropia and myopia. Invest Ophthalmol Vis Sci. 2004;45: 3380-3386.

17. Moriyama M, Ohno-Matsui K, Hayashi K, et al. Topographic analyses of shape of eyes with pathologic myopia by highresolution three-dimensional magnetic resonance imaging. Ophthalmology. 2011;118:1626-1637.

18. Ohno-Matsui K, Akiba M, Modegi T, et al. Association between shape of sclera and myopic retinochoroidal lesions in patients with pathologic myopia. Invest Ophthalmol Vis Sci. 2012;53:6046-6061.

19. Moriyama M, Ohno-Matsui K, Modegi T, et al. Quantitative analyses of high-resolution 3D MR images of highly myopic eyes to determine their shapes. Invest Ophthalmol Vis Sci. 2012;53:4510-4518.

20. Spade RF. Staphyloma: Part 1. New York, NY: Springer; 2013.

21. Ishii K, Iwata H, Oshika T. Quantitative evaluation of changes in eyeball shape in emmetropization and myopic changes based on elliptic Fourier descriptors. Invest Ophthalmol Vis Sci. 2011;52:8585-8591.

22. Lim LS, Chong GH, Tan PT, et al. Distribution and determinants of eye size and shape in newborn children: a magnetic resonance imaging analysis. Invest Ophthalmol Vis Sci. 2013;54:4791-4797.

23. Atchison DA, Pritchard N, Schmid KL, et al. Shape of the retinal surface in emmetropia and myopia. Invest Ophthalmol Vis Sci. 2005;46:2698-2707.

24. Gilmartin B, Nagra M, Logan NS. Shape of the posterior vitreous chamber in human emmetropia and myopia. Invest Ophthalmol Vis Sci. 2013;54:7240-7251.

25. Ohno-Matsui K. Proposed classification of posterior staphylomas based on analyses of eye shape by three-dimensional magnetic resonance imaging and wide-field fundus imaging. Ophthalmology. 2014;121:1798-1809.

26. Gao LQ, Liu W, Liang YB, et al. Prevalence and characteristics of myopic retinopathy in a rural Chinese adult population: the Handan Eye Study. Arch Ophthalmol. 2011;129: 1199-1204.

27. Chang L, Pan CW, Ohno-Matsui K, et al. Myopia-related fundus changes in Singapore adults with high myopia. Am J Ophthalmol. 2013;155:991-999.e1.

28. Wang NK, Wu YM, Wang JP, et al. Clinical characteristics of posterior staphylomas in myopic eyes with axial length shorter than 26.5 millimeters. Am J Ophthalmol. 2016;162: 180-190.e1.

29. Saka N, Ohno-Matsui K, Shimada N, et al. Long-term changes in axial length in adult eyes with pathologic myopia. Am J Ophthalmol. 2010;150:562-568.e1.

30. Miyake M, Yamashiro K, Akagi-Kurashige Y, et al. Analysis of fundus shape in highly myopic eyes by using curvature maps constructed from optical coherence tomography. PLoS One. 2014;9:e107923.

31. Hayashi M, Ito Y, Takahashi A, et al. Scleral thickness in highly myopic eyes measured by enhanced depth imaging optical coherence tomography. Eye. 2013;27:410-417.

32. Jonas JB, Xu L. Histological changes of high axial myopia. Eye. 2014;28:113-117.

33. Jonas JB, Jonas SB, Jonas RA, et al. Histology of the parapapillary region in high myopia. Am J Ophthalmol. 2011;152: 1021-1029.

34. Ohno-Matsui K. What is the fundamental nature of pathologic myopia? Retina 2016 Oct 11 [Epub ahead of print]. 


\section{Footnotes and Financial Disclosures}

Originally received: July 26, 2016.

Final revision: January 3, 2017.

Accepted: January 3, 2017.

Available online: February 22, 2017.

Manuscript no. 2016-1558.

${ }^{1}$ State Key Laboratory of Ophthalmology, Zhongshan Ophthalmic Center, Sun Yat-sen University, Guangzhou, China.

${ }^{2}$ Department of Radiology, The Affiliated Brain Hospital of Guangzhou Medical University (Guangzhou Huiai Hospital), Guangzhou, China.

${ }^{3}$ Research School of Biology, Australian National University, Canberra, ACT, Australia.

${ }^{4}$ Centre for Eye Research Australia, University of Melbourne, Royal Victorian Eye and Ear Hospital, East Melbourne, Australia.

*Xinxing Guo and Ou Xiao made equal contributions to this study.

Financial Disclosure(s):

The author(s) have no proprietary or commercial interest in any materials discussed in this article.

Supported by the Fundamental Research Funds of the State Key Laboratory, National Natural Science Foundation of China (81420108008 and 81271037), Science and Technology Planning Project of Guangdong
Province (2013B20400003), and a grant from the Brien Holden Vision Institute.

Author Contributions:

Conception and design: Guo, Chen, Chen, Morgan, He

Data collection: Xiao, Chen, Wu, Chen

Analysis and interpretation: Guo, Xiao, Chen, Morgan, He

Obtained funding: Not applicable

Overall responsibility: Guo, Xiao, Morgan, He

Abbreviations and Acronyms:

$\mathbf{A L}=$ axial length; $\mathbf{B C V A}=$ best-corrected visual acuity; $\mathbf{D}=$ diopters MRI = magnetic resonance imaging; $\mathbf{O R}=$ odds ratio; $\mathbf{S E R}=$ spherical equivalent refraction; 3D $=$ 3-dimensional; ZOC-BHVI $=$ Zhongshan Ophthalmic Center-Brien Holden Vision Institute.

Correspondence:

Mingguang $\mathrm{He}, \mathrm{MD}, \mathrm{PhD}$, Department of Preventive Ophthalmology, Zhongshan Ophthalmic Center, Sun Yat-sen University, Guangzhou 510060, People's Republic of China. E-mail: mingguang_he@yahoo.com.

\section{Pictures \& Perspectives}
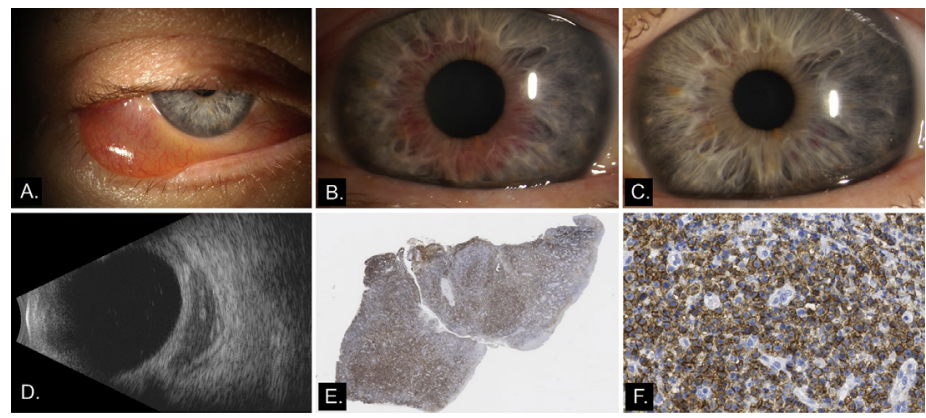

Orbital and Intraocular Lymphoma in Contralateral Eyes: A Case of Cutaneous Aggressive CD-8 + T-Cell Lymphoma

A 63-year-old man presented with right eye swelling 3 months after onset of an ulcerative sacral lesion. Examination of the right eye revealed ptosis, chemosis, proptosis, dysmotility, and a retrobulbar mass on ultrasound (Fig 1A, D). The left eye showed an iris lesion along the pupillary margin (Fig 1B), endothelial keratic precipitates, and anterior chamber cell. Positron emission tomography showed hypermetabolic activity involving sacrum, kidneys, liver, maxillary sinus, hard palate, and right orbit. Biopsy and immunohistochemistry with anti-CD8 antibodies of sacral and oral lesions demonstrated a cutaneous aggressive CD8 + T-cell lymphoma (Fig 1E, 1.25× magnification; Fig 1F, 40× magnification). The patient's ocular signs resolved with chemotherapy (Fig 1C); his treatment is ongoing.

Christopher Komanski, MD, MSE

Kenneth Weinlander, MD

PATRICK YeATTS, MD

Department of Ophthalmology, Wake Forest Baptist Health, Winston-Salem, North Carolina 